\title{
Materiales multimedia: Diseño desde una pedagogía inclusiva
}

\author{
María Esther Baños García, Fernando Lezcano Barbero y \\ Raquel Casado Muñoz \\ Universidad de Burgos (España)
}

\begin{abstract}
La meta de una Competencia Digital Docente (CDD) adecuada está cada vez más cerca para muchos profesionales de la Educación en España. Pensamos que a esta meta habría que añadirle la "I" de Inclusiva (CDDI), promoviendo TICs de diseño y empleo universal que favorezcan ambientes inclusivos en cualquier escenario. En este contexto, nuestro trabajo describe la experiencia desarrollada conjuntamente en dos materias de perfil educativo: Diseño Pedagógico de Materiales Multimedia y Recursos de Comunicación y Adaptación a Usuarios. Mediante la coordinación de su profesorado se buscan dos metas: 1) favorecer aprendizajes más efectivos, económicos en el tiempo del estudiante y reales entre nuestros alumnos que deben trabajar las competencias de ambas materias en coordinación y cooperación y 2) fomentar el aprendizaje reflexivo y creativo en el manejo de estas herramientas. Para ello se trabaja, desde el ejemplo del propio profesorado, empleando aprendizajes activos, el Diseño Universal de aplicaciones multimedia que favorezcan la presencia normalizada de estos materiales a la hora de diseñar planes y curriculums. Atendiendo a las respuestas de los estudiantes a las encuestas docentes, la mayoría están muy satisfechos y, aunque reconocen que les exige un esfuerzo mayor que en otras asignaturas, lo creen muy importante, motivador y altamente demandado para su futuro perfil profesional.
\end{abstract}

\section{Palabras clave: TIC, multimedia, inclusión, DDC (Competencia Digital Docente).}

Multimedia materials: design from an inclusive Pedagogy. The goal of an appropriate Digital Teaching Competence (DTC) is getting closer for many educational professionals in Spain. We think that the "I" of Inclusive should be added to this goal (DITC), in order to promote ICTs of universal design and use that foster inclusive environments at any setting. In this context, our work describes the experience developed jointly in two subjects of educational profile: Pedagogical Design of Multimedia Materials and Communication Resources and Adaptation to Users. By means of the teachers' coordination, two main objectives are sought: 1) to promote more effective, real and time-saving learnings among our students who must work on the competences of both subjects in coordination and cooperation and 2) to encourage reflective and creative learnings in the handling of these tools. To do this, we work, from the example of the teachers themselves, by using active learning, the Universal Design of multimedia applications that foster the normalized presence of these materials when designing plans and curriculums. According to the answers of the students to the educational surveys, the majority are very satisfied and, although they recognize the demanding of a greater effort than in other subjects, they consider it very important, motivating and highly demanded for their future careers.

Keywords: ICT, multimedia, inclusion, DTC (Digital Teaching Competence).

Correspondencia: María Esther Baños García. Escuela Politécnica Superior (Campus Vena). Área de Expresión Gráfica en la Ingeniería. Universidad de Burgos. Avda. Cantabria s/n. C.P.: 09006. Burgos (España). E-mail: ebanyos@ubu.es 
La meta de una Competencia Digital Docente (CDD) adecuada está cada vez más cerca para muchos profesionales de la Educación en España (Tourón, Martín, Navarro-Asencio, Pradas, e Iñigo, 2018). Pensamos que a esta meta habría que añadirle la "I" de Inclusiva (CDDI), promoviendo TICs de diseño y empleo universal que favorezcan ambientes inclusivos, tanto en las aulas como en cualquier otro escenario.

No obstante, la CDD tiene un tratamiento desigual en los planes de estudio de las diferentes universidades españolas (Perez-Escoda, 2015; Sánchez, Alonso, Camacho, y Estebanell, 2017; Sancho-Gil, Bosco-Paniagua, Alonso-Cano, y Sánchez-Valero, 2015). Un análisis de artefactos y documentos (planes docentes y programas de asignaturas) de las Facultades de Educación de un buen número de universidades españolas, y varios estudios (Lores-Gómez, 2017; Pérez-Escoda, 2015; Sánchez, Alonso, Camacho, y Estebanell, 2017; Sancho-Gil, Bosco-Paniagua, Alonso-Cano, y Sánchez-Valero, 2015) nos indica que hay carencias en relación a la CDD (Fernández-Batanero y Rodríguez Martín, 2017) y que es necesario actualizar el modelo de formación inicial de algunas universidades, aún más centradas en contenidos y no tanto en experiencias innovadoras.

En la Facultad de Educación de la Universidad de Burgos venimos apostando por una sólida formación inicial docente integral en TIC, reforzando este tipo de materias, tanto en el número de asignaturas relacionadas, - en el grado de Pedagogía impartimos la Mención Calificadora “Tecnología y Educación”-, como en su impartición, desarrollo, prácticas aplicadas, y la transversalidad de estas competencias en otras asignaturas no específicas (Ausín, Abella, Delgado, y Hortigüela, 2016).

El manejo de recursos multimedia (audio, imagen, video convencional, video interactivo, herramientas de autor) por parte de profesores y alumnos es parte fundamental en esta especialización pues, como señala Pavón (2013) “el entrenamiento en diseño, selección y uso de los medios genera actitudes positivas hacia ellos y los individuos enseñados con determinados recursos se encuentran más fácilmente inclinados a hacer uso de los mismos en su futuro desempeño profesional”.

\section{Objetivos}

En este trabajo se presenta la experiencia desarrollada conjuntamente en dos materias del Grado de Pedagogía de la Universidad de Burgos: Diseño Pedagógico de Materiales Multimedia y Recursos de Comunicación y Adaptación a Usuarios. Mediante la coordinación de su profesorado se buscan dos objetivos: 1) favorecer aprendizajes más eficientes entre nuestros alumnos dado que deben trabajar las competencias de ambas materias en coordinación y cooperación y 2) fomentar el aprendizaje reflexivo y creativo en el manejo de estas herramientas. 


\section{MÉTODO}

Las guías docentes de estas asignaturas son el punto de partida de la coordinación entre el profesorado, elemento primordial de la innovación y mejora educativa (Krichesky y Murillo, 2018). En ellas se ha tratado de incluir las competencias que mejor se ajustan a los objetivos de cada asignatura sin perder la perspectiva más holística de un profesional de la educación. Como ejemplo, cuatro competencias comunes a ambas materias son: 1. Diseñar planes, programas, proyectos, acciones y recursos adaptados a los distintos niveles del sistema educativo, en las modalidades presenciales y virtuales; 2. Diseñar planes de formación del profesorado, de formadores y de otros profesionales, adecuados a las nuevas situaciones, necesidades y contextos; 3. Asesorar sobre el uso pedagógico e integración curricular de los medios didácticos; y 4. Analizar, diseñar y evaluar las aplicaciones de las tecnologías de la información y la comunicación asociadas a los procesos educativos y formativos.

Comenzamos con reuniones previas del profesorado en las que, desde el principio y teniendo presentes las competencias de ambas materias, se preparan el/los proyecto/s-trabajo/s que se llevarán a cabo a lo largo del curso, aunque mantenemos la puerta abierta a alguna posible sugerencia concreta de los alumnos. Los profesores aportamos aquellos proyectos que se nos hayan sugerido externamente (institución, empresa, etc.) o preparamos nuevos según necesidades detectadas en nuestro entorno desde un enfoque de modelo ApS (Aprendizaje-Servicio) (Jouannet, Salas, y Contreras, 2013; Mendía-Gallardo, 2016). A continuación, se prepara un guion básico común, flexible y discutible desde el primer día de clase. De esta forma los estudiantes pueden empezar a documentarse (público diana, objetivos, marco o escenarios) e incluso empezar a recabar materiales que luego incorporarán (audios y/o vídeos por ejemplo de entrevistas, presentaciones, imágenes en forma de fotos que ellos mismos van a tomar o elaboradas a partir de distintas fuentes, etc).

Estos primeros pasos constituyen la primera fase de una metodología general que adapta el modelo tradicional ABP (Aprendizaje Basado en Proyectos) de Maastricht (Schmidt, 1983) adecuado para grupos de 20 a 40 alumnos, que organiza sus siete pasos en tres fases: una primera, donde de forma sincrónica, se trabaja en el aula identificando, definiendo el problema, justificando, preparando la información adicional que se necesitará e identificando los temas sobre los que se va a aprender; una segunda fase, caracterizada por el trabajo más individual de los alumnos; y la tercera, donde se presenta el resultado de los trabajos, generalmente de forma oral, en clase (Prieto, Díaz, Hernández, y Lacasa, 2008; Red de Innovación docente en ABP, 2012). Hablamos de adaptación porque la flexibilidad siempre está presente en nuestra aula $\mathrm{y}$, como indican Travieso Valdés y Ortiz Cárdenas (2018), todas las variantes de los modelos ABP parten de 
entender el problema, analizarlo, establecer objetivos, construir la solución hasta la realización del informe y presentación de los resultados.

En este punto, como transición y paso a la fase dos, comenzamos el desarrollo de los contenidos a la par que van surgiendo las necesidades del trabajo.

Primero sobre manejo de audio donde, con el software libre Audacity, aprenden a grabar (entrevistas, audios para aquellos colectivos con problemas de lectoescritura o visión...), editar y retocar, mejorar audios con ruidos y distorsiones y, finalmente, cómo dejar estos archivos de audio listos para su empleo específico posterior. Churches (2009), enmarca estas herramientas entre las habilidades de pensamiento "Comprender" "Aplicar" y "Crear" y defiende que, en su trabajo de grabación y edición de audios, los estudiantes realmente están construyendo su propio conocimiento.

En segundo lugar, y con criterios similares, se trabajan las imágenes, comenzando por el diseño reflexivo y creativo de una imagen corporativa (logotipo, gama de colores, distribuciones en pantalla) e incluyendo de forma obligatoria la elaboración o empleo de pictogramas y su inclusión como imágenes.

Aunque en todos los puntos es importante, quizás en este y en el punto final donde se incorporan todos los recursos con el programa autor, resulta crucial aplicar varios de los 12 principios de Mayer (Mayer, 2010) básicos cuando se trabaja en entornos digitales multimedia y se intenta integrar las TIC de forma inclusiva y consistente en un marco pedagógico: Principio de coherencia: aprendemos mejor cuando se excluyen palabras, imágenes y sonidos irrelevantes; Principio de señalización: se aprende mejor cuando se "señala" o destaca la información verdaderamente esencial (palabras en negrita, subrayados, exposición en forma de esquema, rodear las imágenes importantes...); Principio de redundancia: valorar la sobreexposición de texto, narración de voz y gráficos para prevenir la sobrecarga cognitiva pues puede ser recomendable en casos de NEEs o contraproducente si no hay alumnos con NEEs, por ejemplo; Principio de contigüidad espacial: se aprende mejor cuando las palabras y sus imágenes correspondientes se muestran cerca unas de otras en la pantalla; Principio de contigüidad temporal: los textos y sus imágenes correspondientes deben presentarse al mismo tiempo, y no de forma sucesiva; Principio de segmentación: el mensaje multimedia debe presentarse en segmentos que el usuario pueda procesar a su ritmo y no como una unidad continua; Principio de pre-formación: si conocemos de antemano las características y los nombres de los conceptos principales, se favorece el aprendizaje multimedia; Principio de Modalidad: aprendemos mejor con imágenes y narración que con animación y texto, pues se limita la sobrecarga cognitiva; Principio Multimedia: se aprende con más facilidad si se presentan palabras e imágenes (estáticas), en vez de solo palabras; Principio de personalización: la información se debe presentar en un lenguaje más coloquial que formal, acorde a la audiencia previsible; Principio de voz: tiene que ver con el tono de la narración y resalta la importancia de la voz humana, frente a la voz sintética; y Principio 
de la imagen: si se graba nuestra voz para un videotutorial, no es necesario incluir nuestra $\mathrm{u}$ otra cara.

En el punto siguiente se trabaja el vídeo convencional, inicialmente con el software Adobe Premiere, aunque si los estudiantes conocen o manejan otro software con similares prestaciones también se puede emplear, ya que lo importante son las tareas obligatorias: realizar vídeo/s de lengua de signos, un croma y saber editar videos que ellos mismos graben (recortar y pegar clips, incluir imágenes, subtitulados, etc).

A continuación, se trabaja el vídeo interactivo, aprendiendo primero a realizar las fotos y a coserlas después para obtener las panorámicas, que posteriormente conformarán los tours virtuales interactivos. De esta forma, pueden incluir la navegación espacial en su proyecto, donde este lo requiera. Empleamos el pack Autopano (realización de panorámicas) y Panotour (para los tours virtuales) que, si bien no es gratuito, sí proporciona una versión "demo" que no caduca y se puede emplear ilimitadamente con la única desventaja de la marca de agua que deja, y que los estudiantes eliminan editando la versión final en clase donde tenemos las licencias oficiales. Tiene también la ventaja de que permite exportar sus proyectos en varios formatos, lo que facilita luego su incorporación o enlace como parte del proyecto.

Aunque se puede pensar en usar siempre software libre (SL), actitud que tratamos de inculcar a nuestros alumnos, también debemos tener en cuenta que no siempre existe la aplicación SL que responde a todas las demandas de nuestros proyectos. Como señalan Serrano y Narváez (2010, p. 49) "la respuesta está en saber integrar los esfuerzos alrededor de las intenciones educativas de las propuestas académicas y no en los medios a utilizar".

El último punto cronológico del proyecto, como resulta lógico, consiste en la incorporación de todos los recursos elaborados en las cuatro fases previas mediante un programa de autor que sea de fácil acceso para los estudiantes y que origine archivos con un formato que puedan llegar a la máxima audiencia. ¿Quién no tiene ya Adobe Reader o trabaja con archivos pdf de una u otra forma? Esta es la razón por la que, aunque existe una gran variedad de programas autor libres y de pago, empleamos Adobe Acrobat, además de por su sencillez de aprendizaje y porque nuestra Universidad nos la proporciona a estudiantes y profesores.

En esta cuarta fase del proyecto se revisan los principios básicos del Diseño Universal para el Aprendizaje (DUA) (Alba-Pastor, 2012; CAST, 2011) en dos vías: en el ámbito interactivo, es decir que cumpla con los requisitos de accesibilidad universal en cuanto a contenidos accesibles, interface, tamaños y tipos de fuentes, etc. (W3C, 2015) para que cualquiera pueda acceder a estos recursos; y en otros ámbitos del DUA, procurando que nuestros materiales cumplan con varias de sus pautas (CAST, 2011) y sirvan así de apoyo en diferentes escenarios inclusivos. Así mismo, se tienen en cuenta la 
metodología de la lectura fácil para facilitar el acceso cognitivo de personas con dificultades lectoescritoras (García-Muñoz, 2014).

\section{RESULTADOS}

Combinando en muchos casos, aprendizaje intencional y servicio a la comunidad, como ocurre en la metodología ApS (Teijeira-Bautista, 2016) obtenemos materiales como guías pedagógicas con distintas funciones, protocolos, presentaciones, todos ellos interactivos, multimedia e inclusivos. Son trabajos reales desarrollados por los alumnos, no solo para las propias asignaturas, sino también en sus TFG, colaboraciones con instituciones, empresas, etc.

Las figuras 1, 2 y 3 muestran ejemplos de materiales elaborados que incluyen una buena representación de los recursos trabajados con las distintas herramientas.

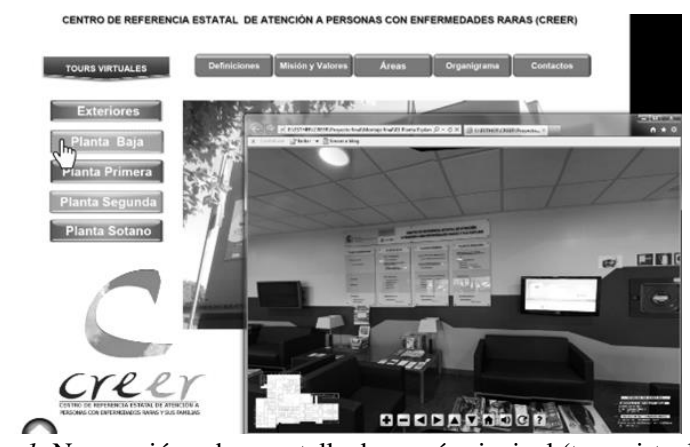

Figura 1. Navegación sobre pantalla de menú principal (tour virtual por el Hall)

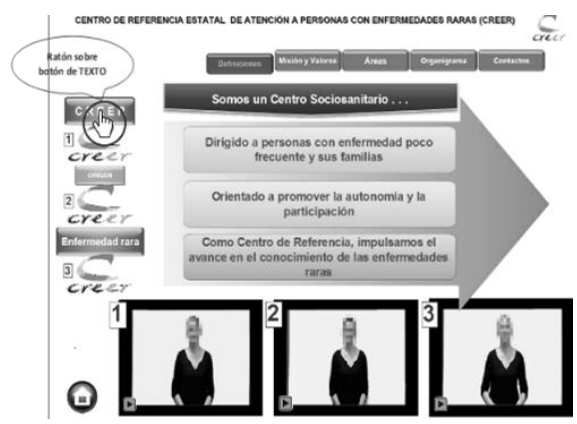

Figura 2. Si no hay problemas de lectura se clica este botón con la definición en texto 


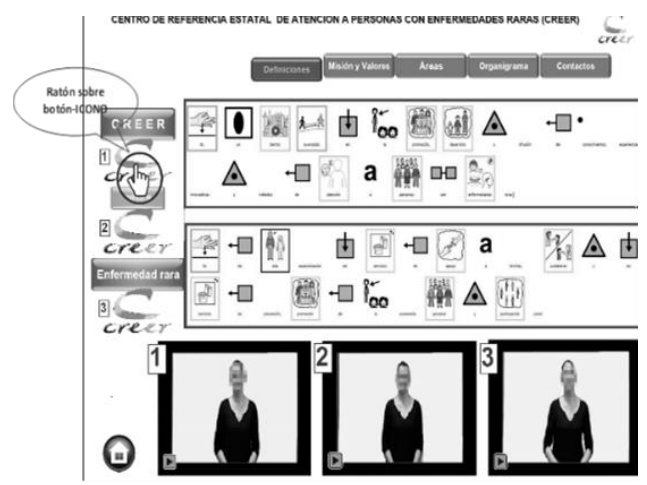

Figura 3. Si hay audiencia que lo requiere, se sitúa el ratón en el botón-icono para ver la definición en pictogramas, o se visualiza el vídeo correspondiente con lengua de signos y audio

Como valoración de la satisfacción y efectividad de este trabajo colaborativo hemos atendido a las respuestas a las encuestas docentes que la Universidad de Burgos realiza cada curso dentro de su plan de Evaluación Docente para su profesorado. En estas encuestas se pregunta a los estudiantes, a final de curso, pero antes de la realización de los exámenes para evitar respuestas sesgadas por los resultados en los mismos acerca de su satisfacción con el desarrollo de la asignatura y la labor de cada profesor.

El número de estudiantes varía cada año, en una parte importante, por ser asignaturas optativas y específicas para un itinerario. Los resultados de participación en la encuesta se recogen en la tabla 1.

Tabla 1. Respuesta del alumnado en la evaluación

\begin{tabular}{cccccc}
\hline Curso & $2012 / 2013$ & $2013 / 2014$ & $2014 / 2015$ & $2015 / 2016$ & $2016 / 2017$ \\
\hline Respuestas & 22 & 10 & 7 & 12 & 12 \\
\hline Matrículas & 26 & 13 & 11 & 17 & 25 \\
\hline$\%$ & $84.6 \%$ & $76.9 \%$ & $63.6 \%$ & $70.6 \%$ & $48 \%$ \\
\hline Fuente: Elaboración propia & & & &
\end{tabular}

Encontramos una respuesta elevada pues la tasa está por encima de $60 \%$, excepto en el curso 2016/17.

Las 6 dimensiones que se valoran, siempre en una escala Likert de 1 a 5 puntos, son: A) Motivación del alumno por la asignatura; B) Materiales de la asignatura; C) Evaluación continua; D) Percepción del estudiante de la motivación del/de la profesor/a hacia la asignatura; E) Percepción de los estudiantes de la carga de trabajo de la asignatura; y F) Satisfacción general con la labor docente de este/a profesor/a, dimensión esta última nueva desde el curso 14/15. En el gráfico 1 recogemos los resultados obtenidos en los últimos cinco años. 
Gráfico 1. Valoraciones de cada dimensión por cursos. Fuente: Elaboración propia

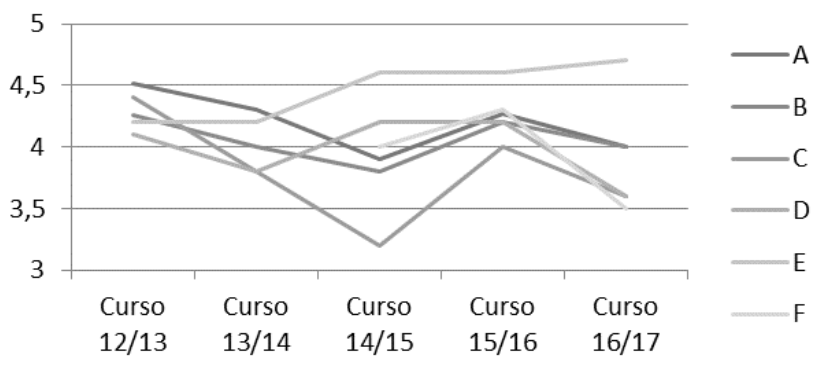

En un análisis descriptivo inicial de estos valores se puede observar cómo las medias están entre el 3.2 y el 4.7 y por encima de la media del resto de las asignaturas impartidas en Pedagogía.

A continuación, analizamos cada una de las dimensiones por separado dadas las importantes diferencias en las características que las componen:

- A. Motivación del alumnado por la asignatura. Las puntuaciones varían entre 4 y 4.5 teniendo como media 4.2. Esto pone de manifiesto la elevada motivación con la que cuentan los estudiantes. Dado que, como hemos indicado, son asignaturas optativas y específicas de la Mención "Tecnología y Educación”, debemos entender que las asignaturas implicadas son acordes con los intereses del alumnado participante.

- B. Materiales de la asignatura. Las puntuaciones se encuentran entre 3.8 y 4.3, tiene como media 4.05. Como en el caso anterior los materiales (información aportada sobre el software, uso...) se reconoce como recursos o herramientas de alto valor.

- C. Evaluación continua. Las puntuaciones varían entre 3.6 y 4.4, teniendo como media 3.8. Aunque la puntuación media es igual a la dimensión anterior, encontramos una mayor variabilidad que se centra en las valoraciones del curso 14/15. Cuando se detectó este descenso, se analizaron sus causas entre profesores y también en el seguimiento que anualmente se hace en las reuniones de coordinación de curso y de titulación. Si bien en las reuniones de coordinación se comentó acerca de las diferencias entre los alumnos de este curso y los de otros previos, los profesores también concluimos, que los trabajos valorados en la evaluación continua habían supuesto un sobre esfuerzo a este grupo, razón por la que se intentaron ajustar mejor para los próximos cursos. Además, como ocurre en el curso 16/17, las respuestas a la encuesta también bajaron en porcentaje respecto a los matriculados, hecho que también pudo influir en este descenso.

- D. Percepción del estudiante de la motivación del/de la profesor/a hacia la asignatura. Las puntuaciones varían entre 3.6 y 4.2, teniendo como media 3.98. Esta 
puntuación positiva, muy cerca del 4 , nos sorprende pues creemos que la motivación y esfuerzo del profesorado no se corresponde con la percepción del alumnado.

- E. Percepción de los estudiantes de la carga de trabajo de la asignatura. Las puntuaciones varían entre 4.2 y 4.7, teniendo como media 4.5. Es importante apuntar la carga que supone esta asignatura respecto a otras con el mismo número de créditos y reconocemos que es elevada. En general, cuando los alumnos comienzan, reconocen hacerlo con temor, debido a que su competencia digital, sobre todo en lo que se refiere a manejo de softwares especializados, es bastante limitada, detectando una gran mejora en el semestre que la cursan, pero a costa de un considerable esfuerzo.

- F. Satisfacción general con la labor docente (desde el curso 14/15). Las puntuaciones varían entre 3.5 y 4.3 , teniendo como media 3.9.

Con respecto a la caída de algunas medias en los cursos 14/15 y 16/17, se puede observar que son los cursos donde, por razones diversas (visitas realizadas en otras asignaturas, finalización de proyectos en fechas muy próximas a exámenes donde la asistencia a clase se resiente, autoexigencia de obtener buenos resultados para presentar a los centros, etc.), los encuestados representaron un porcentaje mucho menos representativo respecto a los matriculados.

En suma, encontramos que la percepción del alumnado respecto del trabajo del profesorado (búsqueda de proyectos, apoyo al aprendizaje de aplicaciones, resolución de dificultades técnicas...) no siempre se valora como podríamos desear.

En estos aspectos encontramos los puntos de mejora en que trabajamos actualmente. Una de las líneas, como ya se ha explicado en puntos anteriores, es realizar proyectos que comprendan las competencias en este caso de dos asignaturas, para economizar así tiempos de trabajo de los estudiantes, y por otro tratando de ajustar los proyectos y su desarrollo, bien simplificando algunos complejos, o bien desglosándolos en partes que puedan luego reunirse sin perder el objetivo holístico del proyecto/trabajo. Aquí son importantes los acuerdos de todos sobre la planificación del proyecto que debe dejarse bien enmarcada, detallada y clara.

\section{DISCUSIÓN Y CONCLUSIONES}

Inmersos ya en pleno siglo XXI, y con evidencias de que la Competencia Digital Docente es una realidad para muchos de los profesionales de la educación en nuestro país (Tourón, Martín, Navarro-Asencio, Pradas, e Iñigo, 2018) parece el momento de seguir avanzando en esta línea añadiendo a estas competencias otros retos que normalicen prácticas TIC considerando la Pedagogía Inclusiva (Florian y Beaton, 2018). En nuestras titulaciones de la Facultad de Educación de la Universidad de Burgos, tanto sus profesores como sus alumnos, a los que ya podemos calificar de nativos digitales, no solo somos conscientes de estos retos (Sánchez i Valero, Guitert-Catasús, Rivera-Vargas, 
y Simón-Llovet, 2017) sino que el nivel de implicación de ambos colectivos es importante, como lo demuestran cada año las nuevas propuestas metodológicas en distintas asignaturas, tecnológicas o no (p.e. Delgado, Hortigüela, Ausín, y Abella, 2017) y la respuesta de los estudiantes quienes, cada vez más, eligen el itinerario de "Tecnología y Educación”, conscientes de la demanda que ya detectan y de su importancia para el futuro ejercicio de la profesión educativa.

Las cifras encontradas en nuestro estudio nos indican que debemos seguir trabajando en el desarrollo metodológico coordinado de estas materias haciendo énfasis en prácticas TAC (Tecnologías para el Aprendizaje y la Comunicación) que mejoren la eficacia y reduzcan el esfuerzo que debe hacer el estudiante, siempre sin perder de vista las competencias específicas y transversales a adquirir dentro de la Pedagogía Inclusiva.

\section{Agradecimientos}

Este trabajo se enmarca en el Proyecto BU074G18 "Autonomía y derecho a la participación de los niños y jóvenes con necesidades de apoyo socieducativo. Hacia un nuevo paradigma", financiado por la Junta de Castilla y León en la convocatoria de subvenciones destinadas al apoyo a Grupos de Investigación Reconocidos de Universidades Públicas de Castilla y León 2018-2021.

\section{REFERENCIAS}

Ausín, V., Abella, V., Delgado, V., y Hortigüela, D. (2016). Aprendizaje Basado en Proyectos a través de las TIC. Una experiencia de Innovación Docente desde las Aulas Universitarias. Formación Universitaria, 9(3), 31-38. doi: 10.4067/S0718-50062016000300005.

Fernández-Batanero, J.M., y Rodríguez Martín, A. (2017). TIC y diversidad funcional: conocimiento del profesorado. European Journal of Investigation in Health, Psychology and Education, 7(3), 157-175. doi:10.30552/ejihpe.v7i3.204.

CAST (2013). Pautas sobre el Diseño Universal para el Aprendizaje (DUA). Versión 2.0 (Carmen Alba Pastor, Pilar Sanchez Hipola, José Manuel Sanchez Serrano y Ainara Zubillaga del Río, traduc.). Universidad Complutense de Madrid. (Obra original publicada en 2011).

Churches, A. (2009). Taxonomía de Bloom para la era digital. Recuperado de: http://eduteka.icesi.edu.co/articulos/TaxonomiaBloomDigital

Delgado, V., Hortigüela, D., Ausín, V., y Abella, V. (2017). Evaluación formativa a través de metodologías activas: Proyecto RadioEDUBU. Revista Infancia, Educación y Aprendizaje, 3(2), 19-24.

Florian, L., y Beaton, M. (2018). Inclusive pedagogy in action: getting it right for every child. International Journal of Inclusive Education, 22(8), 870-884.

García, Ó. (2014). Lectura fácil: métodos de redacción y evaluación. Madrid: Real Patronato sobre Discapacidad.

Herrada, R.I., y Herrada, G. (2011) Adaptación de los estudios de Magisterio al EEES: las TIC en los nuevos planes de estudio. Edutec-e, Revista Electrónica de Tecnología Educativa, 36. Recuperado de: http://edutec.rediris.es/revelec2/revelec36 
Jouannet, C.H., Salas, M. H., y Contreras, M.A. (2013). Modelo de implementación de Aprendizaje Servicio $(\mathrm{A}+\mathrm{S})$ en la UC: Una experiencia que impacta positivamente en la formación profesional integral. Calidad en la Educación, (39), 197-212. doi: 10.4067/S071845652013000200007.

Krichesky, G.J., y Murillo, F.J. (2018). La colaboración docente como factor de aprendizaje y promotor de mejora: Un estudio de casos. Educación XXI, 21(1), 135-156. doi: 10.5944/educXX1.15080.

Lores, N. (2017). Estudio descriptivo del uso de las TIC en Educación Primaria como respuesta a la realidad educativa y social en la provincia de Castellón (Tesis de doctorado inédita). Universidad CEU Cardenal Herrera, Valencia, España.

Mayer, R.E. (2010). Multimedia Learning. Cambridge, New York: Cambridge University Press.

Mendía-Gallardo, R. (2016). El aprendizaje-servicio: una metodología para la innovación educativa. Convives: Aprendizaje-Servicio y Convivencia, (16), 20-26.

Pastor, C. (2012). Aportaciones del Diseño Universal para el Aprendizaje y de los materiales digitales en el logro de una enseñanza accesible. Recuperado de: http://diversidad. murciaeduca. es/publicaciones/dea2012/docs/calba. pdf.

Pavón Ravasco, F. (2013). La introducción de las TIC en el curriculum y en la organización escolar de la educación infantil y primaria. Buenas Prácticas Educativas en el uso de las TIC, Volumen 1. Jaén (España): Joxman Editores, S.L.

Pérez-Escoda, A. (2015). Alfabetización Digital y Competencias Digitales en el Marco de la Evaluación Educativa: Estudio en docentes y alumnos de Educación Primaria en Castilla y León (Tesis de doctorado inédita). Salamanca: Departamento de Didáctica, Organización y Métodos de Investigación. Universidad de Salamanca.

Prieto, A., Díaz, D., Hernández, M., y Lacasa, E. (2008). Variantes metodológicas del ABP: el ABP 4x4. En J. García Sevilla (Coord.), El aprendizaje basado en problemas en la enseñanza universitaria. (pp. 55-74). Murcia: Universidad de Murcia. Servicio de Publicaciones.

Red de Innovación Docente en ABP del ICE de la Universidad de Girona. (2012). El ABP: origen, modelos y técnicas afines. Aula de Innovación Educativa, (216), 14-18.

Sánchez, J.A., Alonso, C., Camacho, M., y Estebanell, M. (2017). Análisis de la presencia de las Competencias Digitales Docentes en los planes de estudio de los grados de maestro en universidades catalanas. V Congreso Internacional de Docencia Universitaria CINDU: Vigo (España).

Sánchez, J.A., Guitert, M., Rivera, P., y Simón, J. (2017). Visiones de maestros y estudiantes sobre la formación inicial en competencias digitales: formación del profesorado en tecnología educativa. XXV Jornadas Universitarias de Tecnología Educativa JUTE: Burgos (España).

Sancho-Gil, J.M., Bosco-Paniagua, A., Alonso-Cano, C., y Sánchez-Valero, J.A. (2015). Formación del profesorado en Tecnología Educativa: de cómo las realidades generan los mitos. Revista Latinoamericana de Tecnología Educativa RELATEC, 14(1), 17-30. doi: 10.17398/1695288X.

Schmidt, H.G. (1983). Problem-based learning: rationale and description. Medical Education 17, 11-16. doi: 10.1111/j.1365-2923.1983.tb01086.x.

Serrano, J.E., y Narváez, P.S. (2010). Uso de Software Libre para el Desarrollo de Contenidos Educativos. Formación Universitaria, 3(6), 41-50. doi: 10.4067/S071850062010000600006.

Teijeira-Bautista, E. (2016). Aprendizaje-Servicio: una metodología que funciona. Convives: Aprendizaje-Servicio y Convivencia, (16), 5-11. 
Tourón, J., Martín, D., Navarro, E., Pradas, S., e Íñigo, V. (2018). Validación de constructo de un instrumento para medir la competencia digital docente de los profesores (CDD). Revista Española de Pedagogía, 76(269), 25-54. doi: 10.22550/REP76-1-2018-02.

Travieso, D., y Ortiz, T. (2018). Aprendizaje basado en problemas y enseñanza por proyectos: alternativas diferentes para enseñar. Revista Cubana de Educación Superior, (1), 124-133.

W3C (2015). Authoring Tool Accessibility Guidelines (ATAG) 2.0. W3C Recommendation. Recuperado de: https://www.w3.org/TR/ATAG20/

Recibido: 5 de julio de 2018 Recepción Modificaciones: 19 de septiembre de 2018 Aceptado: 2 de noviembre de 2018 